\title{
Behavioral Couples Therapy for Substance Use Disorders: A Model for Implementation
}

\author{
Klostermann $\mathrm{K}^{1 *}$, Papagni $\mathrm{E}^{2}$ and Henninger $\mathrm{MW}^{3}$ \\ ${ }^{1}$ Walden University, USA \\ ${ }^{2}$ Medaille College, USA \\ ${ }^{3}$ University at Buffalo, USA
}

Review Article

Volume 5 Issue 2

Received Date: June 23, 2021

Published Date: August 31, 2021

DOI: $10.23880 / \mathrm{mhrij}-16000148$

*Corresponding author: Keith Klostermann, Walden University, 100 Washington Avenue South, Minneapolis, MN 55401; USA, Email: keith.klostermann@mail.waldenu.edu

\section{Abstract}

The important role of family in the development and maintenance of substance use disorders is now widely acknowledged by researchers and clinicians alike. Behavioral Couples Therapy for Substance Use Disorders (BCT-SUD) is an evidencebased treatment based on social learning theory which posits that couples in which a partner has a substance use disorder are characterized by a reciprocal pattern of unhealthy substance use and negative relationship dynamics that reinforce relationship dysfunction and problematic substance use. Despite BCT-SUD's proven efficacy across several domains of functioning, it is not widely implemented into practice. Therefore, the challenge for BCT-SUD researchers and clinicians alike is to identify comprehensive and systemic models for implementation which are not only feasible, but sustainable. The purpose of this paper is to provide a possible model for implementing BCT-SUD in practice as well as identify common barriers to the implementation process.

Keywords: Behavioral Couples Therapy; Evidence Based Treatment; Disorders

Abbreviations: BCT-SUD: Behavioral Couples Therapy for Substance Use Disorders; FIT: Feedback Informed Treatment.

\section{Behavioral Couples Therapy for Substance Use Disorders: A Model for Implementation}

The critical role of families in the development and maintenance of substance use disorders is now widely acknowledged by researchers and clinicians alike. In fact, previous literature has documented a bidirectional relationship between relationship discord and substance use, such as substance use being a potential response to relationship conflict and conflict may lead to substance use/misuse [1]. To absolve this, the incorporation of family members or significant others in the treatment process has become increasingly common regardless of presenting concern or level of care e.g., outpatient, in-patient; [2]. Among couple and family treatments, Behavioral Couples Therapy for Substance Use Disorders (BCT-SUD) has been extensively studied with results consistently demonstrating superior substance use and relationship outcomes compared to treatment-as-usual i.e., individual-based treatment [3]. BCT-SUD is a conjoint approach which has steadily shown to produce fewer substance-related issues, greater abstinence, and improved dyadic functioning compared to individualbased treatments for married and cohabitating couples [4]. This finding has also been supported by the results of several 


\section{Mental Health \& Human Resilience International Journal}

meta-analyses, which found medium effect sizes favoring the use of family and/or couples therapy relative to other forms of treatment [4]. In addition to improvements in the primary outcomes, patients receiving BCT-SUD also report improvements in secondary domains including intimate partner violence [5], children's adjustment, and cost [6].

BCT-SUD is an evidence-based treatment based on social learning theory, which suggests that couples in which a partner has a substance use disorder are characterized by a reciprocal pattern of unhealthy substance use and negative relational dynamics, which serve to reinforce relationship dysfunction and problematic substance use [7]. This circular pattern of substance misuse and dyadic dysfunction is often referred to a "vicious cycle" with one negative behavior precipitating and reinforcing the other.

The effectiveness of BCT for substance abusing patients and their partners is no longer in question. In fact, Division 50 of the American Psychological Association (Empirically Supported Treatments for Substance Use Disorders Committee) recently reviewed the outcome evidence for BCT for alcohol dependence and concluded this treatment meets the criteria for "well established" treatment, which is the highest level of empirical support recognized by Division 50. Schumm and Renno's [8] review also concluded that BCT-SUD is suitable for real-world clinical settings and can be adapted to meet the needs of the community in which it's being implemented, such as abbreviated formats and telehealth protocols. However, despite its proven efficacy across several different domains of functioning, BCT-SUD has not been widely implemented in practice. In fact, McGovern MP, et al. [9] examined community addiction providers (i.e., Directors [ $n=21]$ and clinicians [ $n=89]$ ) experiences, beliefs, and readiness to implement a variety of evidencebased practices. Results were mixed; providers reported more readiness to adopt twelve-step facilitation, cognitive behavioral therapy, motivational interviewing, and relapse prevention, while less ready to implement contingency management, BCT, and pharmacotherapies. McGovern MP, et al. [9] concluded that in order for treatments to be successfully disseminated, investigators must clearly demonstrate the relevance of the treatment to clinicians and staff, even if empirical support is already established. Thus, the challenge for BCT-SUD researchers and clinicians alike is to identify comprehensive and systemic models for implementation which are not only feasible, but sustainable [8]. The purpose of this paper is to provide a possible model for implementing BCT-SUD in practice as well as identify common barriers to the implementation process. Using Fixsen DL, et al. [10] recommendations for implementation, coupled with the Feedback Informed Treatment (FIT) implementation machinery, we propose a method for introducing and implementing BCT-SUD in practice.

\section{Implementation}

According to Rogers E [11], there are four main criteria which must be considered when considering translating a research program into practice:

- The innovation (e.g., BCT-SUD),

- Communication channels.

- Time (how long?).

- The social system itself.

At a more individual level, Rogers E [11] asserts that an individual's decision to adopt or reject an evidence-based practice is influenced by:

- Relative advantage (i.e., how is the new service better than the old?).

- Compatibility (i.e., how does the new service fit within the current system?).

- Level of complexity or simplicity.

- Trial ability (can the individual test the new service in advance of full implementation?).

- Observability (how visible is the service to others).

- Generally speaking, the goals of any implementation effort are to create change in adult practitioner behavior, revise and refine organizational structure and culture (e.g., values, philosophies, ethics policies, procedures), and change relationships with clients, key stakeholders, and system partners.

\section{Implementation Steps}

Successful implementation requires a methodical and systematic approach which may be best conceptualized as process, rather than a time-limited event, which involves a series of sequential phases. It must be noted, that during the implementation process organizations may slide back into earlier phases based on the practical realities of the organization. In reviewing the implementation science literature, Fixsen DL, et al. [10] identified six stages which seem critical to implementing evidence-based practices:

- Exploration and adoption.

- Installation.

- Initial implementation.

- Full operation.

- Innovation.

- Sustainability.

Each of these stages is described briefly below along with their implications for BCT-SUD.

\section{Exploration}

In this stage, key program personnel are tasked with exploring the match between the needs of the community, the evidence-based treatment and what is involved in delivering it, and the community resources with the goal of making an informed decision about whether to proceed 


\section{Mental Health \& Human Resilience International Journal}

with the implementation or not. Assembling a small group of interested staff willing to introduce the approach to staff and key stakeholders (administrators, supervisors, etc.) and begin to discuss the merits as well as the issues implementation of the new approach may present. Management support is critical to the success of the implementation initiative.

From a BCT-SUD perspective, some of the primary considerations may involve provider attitudes towards couples therapy, management involvement to support the effort and in what ways (including financially), implications for existing policy and procedures. Ultimately, the key questions during this phase involves feasibility.

\section{Installation}

This is typically the costliest stage of the implementation and involves creating structures to support the new treatment, training supervisors in the approach, adjusting productivity standards to accommodate the training efforts, running small pilot implementations, and identifying people who are passionate about the new program and willing to take a risk (i.e., 'champions'). During the installation phase, challenges are the norm and perfection is not expected; rather the goal may be best conceptualized as failing in a survivable manner. The results of these efforts and lessons learned in the pilots can be used to revise existing methods and inform the larger implementation effort. During this phase as well, it is critical that management participates in the training so they are familiar with the scope of the new treatment and have a better understanding of the systemic, clinical, and administrative changes required for successful implementation.

In this stage, it is important to identify excited and enthusiastic BCT-SUD staff to serve as advocates for the program. If not easily identifiable, often during the training these staff emerge as they become more excited about the possibility of implementing a new treatment. The importance of this role cannot be understated - these are the staff that will be critical in garnering support and working through programmatic and procedural obstacles.

\section{Initial Implementation}

The information learned and structures put in place during the first two stages (i.e., exploration and installation) are now applied in practice during the initial implementation. According to Fixsen DL, et al. [10], "during the initial stage of implementation the compelling forces of fear of change, inertia, and investment in the status quo combine with the inherently difficult and complex work of implementing something new" (p. 16). Given the potential ambivalence about change, coupled with the introduction of a new way of operating, Joyce BR, et al. [12] has referred to this stage as the "initial awkward phase." As such, on-going coaching around the new treatment, as well as changes in policy and procedure are often necessary as a way to ensure continued compliance.

This is a time where staff may need to be reminded that the new changes are not optional and require compliance on everyone's part. In the case of BCT-SUD, providers may not be used to conducting couples therapy or even considering it as an option. Administrators and clerical staff may also be unfamiliar with the billing process for the new service. It can also be helpful to examine what is working as well as what may need to modified or refined. During this phase, it is important to highlight and note the successes achieved thus far and communicate this with all staff.

\section{Full Operation}

During this phase there is continued performance monitoring at both the individual and organizational level to incorporate new developments into existing practice and policies in an effort to maintain and improve the new policies and procedures. Management and leadership are expected to problem solve issues as needed and continue efforts to change the culture. At this point, all staff is expected to apply the new treatment which may involve adhering to new policies and procedures. The goals during this stage include full staffing (as related to the new treatment), full client caseloads, active and on-going referrals based on the inclusion/exclusion criteria, practitioner skill in applying the intervention in practice (i.e., adherence and competence in delivering the model), managerial and administrative support for the new policies and practices, and community acceptance of the new service [10]. In other words, the evidence-based treatment becomes part of the treatment as usual milieu.

In this phase, all staff (clerical, providers, and administrators) will be familiar with the BCT-SUD service, identified staff and supervisors will be appropriately trained, providers will be familiar with appropriate referrals, and this service will be fully integrated into the current armamentarium of services. Relatedly, BCT-SUD paperwork will be fully integrated into existing policy, procedure, and record-keeping. During this phase, regular communication among staff about the new service and progress is important to ensure consistency in messaging about the success of the program as well as any changes.

\section{Sustainment}

The goal of this phase involves the long-term survival of the new program within the context of an ever-changing and dynamic environment (e.g., turnover, changes in funding, 


\section{Mental Health \& Human Resilience International Journal}

new practice guidelines). Sustainment is not an endpoint, but may be best viewed as on-going process that involves proactive planning to handle issues and obstacles as they arise in order to ensure continued effectiveness and feasibility.

Given the high rates of turnover among staff and leadership, coupled with limited (or shrinking) resources, it is critical that organizations plan ahead to deal with these issues. In particular, it may be wise to offer on-going training for new staff as part of orientation as well as regularly scheduled boosters for current staff to ensure continued adherence to the program [13].

\section{Common Barriers}

Given the change process can be uncomfortable for many, as well as inertia, implementation problems are inevitable. Consequently, it's highly recommended that practitioners and supervisors are held accountable for their efforts (or lack thereof) rather than the members of the implementation team. In addition, it is also important to work with providers and administrators in tempering their expectations for the implementation process. Many well intentioned and excited providers/agencies interested in implementing a new treatment quickly fizzle out because of unrealistic expectations. It's important to note that implementation is a lengthy process and organizations and their staff are best served by thinking in terms of years, rather than months. In fact, successful implementation efforts may take 3-7 years to reach full implementation and sustainability. Unfortunately, many implementation efforts fail because leaders' planning fallacy (i.e., not having a realistic sense of how long these efforts take). Moreover, other common pitfalls involve the misguided focus practitioner skill in delivering the intervention, rather than organizational change (e.g., policy, procedure) and lack of continuity in staffing (i.e., high turnover rates among leadership and key personnel).

\section{Conclusion}

Although BCT-SUD is an evidence-based treatment that is proven to be effective across several domains of functioning, it is not widely implemented into clinical practice. Therefore, the challenge for BCT-SUD researchers and clinicians alike is to identify comprehensive and systemic models for implementation which are not only feasible, but sustainable. Successful implementation requires a methodical and systematic approach which may be best conceptualized as process, rather than a time-limited event, which involves a series of sequential phase. Fixsen DL, et al. [10] identified six stages that appear critical to implementing evidence-based practices and can be utilized for the implementation of BCTSUD:

- Exploration and adoption,
- Installation.

- Initial implementation.

- Full operation.

- Innovation.

- Sustainability.

Following these six steps offers the implementation process a guided framework for reference as well as a more streamlined process. Given that change can be difficult and uncomfortable, challenges are likely to arise during the implementation process; however, there are strategies to try to minimize barriers.

\section{References}

1. Mason R, O'Rinn SE (2014) Co-occurring intimate partner violence, mental health, and substance use problems: A scoping review. Global Health Action 7(1): 1-17.

2. Klostermann K, O'Farrell TJ (2020) Alcohol and other substance use disorders. In: Wampler K (Ed.), Handbook of Couple, Marital, and Family Therapy: The Couple Subsystem in Couple, Marital, and Family Therapy. $3^{\text {rd }}$ (Vol.), Wiley: UK, pp: 188-202.

3. Klostermann K, Mignone T (2019) Behavioral couples therapy for substance use disorders. Social Behavior Research \& Practice 3(1): 25-27.

4. O'Farrell TJ, Schumm JA, Dunlap LJ, Murphy MM, Muchowski P (2016) A randomized clinical trial of group versus standard behavioral couples therapy plus individually based treatment for patients with alcohol dependence. Journal of Consulting and Clinical Psychology 84(6): 497-510.

5. Easton CJ, Crane CA (2016) Interventions to reduce intimate partner violence perpetration among people with substance use disorders. International Review of Psychiatry 28(5): 533-543.

6. Mignone T, Papagni E, Mahadeo M, Klostermann $\mathrm{K}$, Jones RA (2017) PTSD and intimate partner violence: Clinical considerations and treatment options. Journal of Addiction Medicine and Therapeutic Science 3(1): 1-6.

7. Byrne M, Carr A, Clark M (2004) The efficacy of behavioral couples therapy and emotionally focused therapy for couple distress. Contemporary Family Therapy: An International Journal 26(4): 361-387.

8. Schumm JA, Renno S (2021) Implementing behavioral couples therapy for substance use disorders in realworld clinical practice. Family Process.

9. McGovern MP, Fox TS, Xie H, Drake RE (2004) Evidencebased practices: Dissemination research in an addiction 


\section{Mental Health \& Human Resilience International Journal}

treatment system. Journal of Substance Abuse Treatment 26(4): 305-312.

10. Fixsen DL, Naoom SF, Blase KA, Friedman RM, Wallace $F$ (2005) Implementation research: A synthesis of the literature. Tampa, FL: University of South Florida, Louis de la Parte Florida Mental Health Institute, The National Implementation Research Network, FMHI Publication.

11. Rogers E (2003) Diffusion of Innovations. $5^{\text {th }}$ (Edn.), Free
Press.

12. Joyce BR, Showers B (2002) Student Achievement through Staff Development. $3^{\text {rd }}(\mathrm{Edn})$, Association for Supervision \& Curriculum Deve (ASCD).

13. Jacobson NS, Margolin G (1979) Marital therapy: Strategies based on social learning and behavior exchange principles. Brunner/Mazer Publishers, pp: 424. 